\title{
Mitochondrial Fusion/Fission, Transport and Autophagy in Parkinson's Disease: When Mitochondria Get Nasty
}

\author{
Daniela M. Arduíno, ${ }^{1}$ A. Raquel Esteves, ${ }^{1}$ and Sandra M. Cardoso ${ }^{1,2}$ \\ ${ }^{1}$ Center for Neuroscience and Cell Biology (CNC), University of Coimbra, Largo Marquês de Pombal, \\ 3004-517 Coimbra, Portugal \\ ${ }^{2}$ Faculty of Medicine, University of Coimbra, Largo Marquês de Pombal, 3004-517 Coimbra, Portugal
}

Correspondence should be addressed to Sandra M. Cardoso, smacardoso@yahoo.com

Received 1 October 2010; Revised 26 November 2010; Accepted 5 January 2011

Academic Editor: Charleen T. Chu

Copyright ( 2011 Daniela M. Arduíno et al. This is an open access article distributed under the Creative Commons Attribution License, which permits unrestricted use, distribution, and reproduction in any medium, provided the original work is properly cited.

Understanding the molecular basis of Parkinson's disease (PD) has proven to be a major challenge in the field of neurodegenerative diseases. Although several hypotheses have been proposed to explain the molecular mechanisms underlying the pathogenesis of $\mathrm{PD}$, a growing body of evidence has highlighted the role of mitochondrial dysfunction and the disruption of the mechanisms of mitochondrial dynamics in PD and other parkinsonian disorders. In this paper, we comment on the recent advances in how changes in the mitochondrial function and mitochondrial dynamics (fusion/fission, transport, and clearance) contribute to neurodegeneration, specifically focusing on PD. We also evaluate the current controversies in those issues and discuss the role of fusion/fission dynamics in the mitochondrial lifecycle and maintenance. We propose that cellular demise and neurodegeneration in $\mathrm{PD}$ are due to the interplay between mitochondrial dysfunction, mitochondrial trafficking disruption, and impaired autophagic clearance.

\section{Introduction: The Critical Role of Mitochondrial Dysfunction in PD}

The intrinsic properties of mitochondria make them essential integrators of cellular functions. These organelles are critical as ATP suppliers, calcium buffers and transducers of intracellular signaling pathways which integrate programmed cell death. Therefore, mitochondrial function has a critical role in the brain physiology, where the impaired functioning of mitochondria has been implicated in several neurological disorders, like Parkinson's disease (PD).

$\mathrm{PD}$ is a chronically progressive, age-related neurodegenerative disease, clinically characterized by progressive resting tremor, rigidity, bradykinesia, gait disturbance, postural instability, and dementia. A major neuropathological hallmark of PD is the degeneration of dopaminergic neurons in the substantia nigra pars compacta (SNpc) and in other brainstem regions. A second neuropathological feature is the presence of intracytoplasmic inclusions (Lewy bodies, LBs) in surviving neurons, which comprise a dense core of different proteins as $\alpha$-synuclein, parkin, ubiquitin, synphilin-1, tubulin, and other cytoskeletal proteins [1]. Over the last several decades, a growing body of evidence accumulated focusing on the crucial role of mitochondria and mitochondrial dysfunction in PD etiopathogenesis [2$6]$.

The most compelling evidence of mitochondrial dysfunction in PD emerged following the human accidental exposure to the synthetic meperidine analogue 1-methyl4-phenyl-1,2,3,6-tetrahydrodropyridine (MPTP) which induced a parkinsonian syndrome through a decrease in complex I activity by its metabolite 1-methyl-4-phenylpyridinium $\left(\mathrm{MPP}^{+}\right)[7,8]$.

Furthermore, mitochondrial association with idiopathic PD was first established when a mitochondrial NADH dehydrogenase (complex I) activity deficit was identified in the SNpc of postmortem PD patients brains [9] and in PD patients platelets [10]. Further evidence suggested a similar complex I deficiency in PD patients lymphocytes [11, 12], although few reports failed to demonstrate consistent 
changes between PD and control cells [13]. To address the potential causes of complex I defect, that is, if it was due to an environmental toxin or to an alteration of mitochondrial or nuclear DNA, the cytoplasmic hybrid technique has been used. This approach consists on the transfer of sporadic PD subject platelet mitochondria to mtDNA-depleted cells, generating hybrid lines (cybrids). In this way, it is possible to follow the expression of the mitochondrial mutations and the effects of its heteroplasmy in a wild-type nuclear DNA environment. A stable decrease in complex I activity, increased reactive oxygen species (ROS) production, proton leak, and decreased maximum respiratory capacity were described in PD cybrids [14-17]. All of these features are consistent with the involvement of respiratory chain dysfunction in PD. In addition to mtDNA mutations, the products of familial PD-linked genes, including $\alpha$-synuclein, parkin, DJ-1, PINK1 (PTEN-induced kinase 1), LRRK2 (Leucine-rich repeat kinase 2), were shown to locate in or interact with mitochondria under certain conditions $[18,19]$. In addition, loss of OMI protease activity increases the susceptibility of mitochondria to induce the permeability transition [20]. Moreover, LRRK2 colocalizes with the mitochondrial outer membrane [21] and may regulate the response to mitochondrial inhibitors [22]. Other studies revealed many novel proteins with quantitative expression differences in PD when compared to controls. Those include subunits of complex I, mitochondrial creatine kinase, the chaperone mortalin (mthsp70/GRP75), and glutathione $s$-transferase pi, all proteins implicated in mitochondrial functions and cellular responses to oxidative stress $[23,24]$.

This body of evidence is suggestive that mitochondrial function is altered in PD. In this paper we will focus on mitochondrial dynamics, emphasizing the changes in mitochondrial motility and mitochondrial quality control mechanisms, critical to maintain mitochondrial function and cellular homeostasis. Moreover, we will also address the contributions of these topics to cellular demise associated to PD neurodegeneration and comment on the current controversies that exist on this issue.

\section{Mitochondrial Dynamics and PD}

2.1. Mitochondrial Dynamics. Mitochondria can appear as discrete tubules or interconnected networks in living cells [25]. This range on mitochondrial shaping and size profiles is continuous since the hundreds of mitochondria within a cell can undergo frequent cycles of fusion (the combination of two mitochondria into a single organelle) and fission (the separation of long, tubular mitochondria into two or more smaller parts) $[26,27]$. This fusion/fission dynamics is very important in maintaining the functional integrity of mitochondria as the constituents of each network share solutes, metabolites, and proteins [28-30], as well as electrochemical gradient, making them electrically coupled $[31,32]$. These mitochondrial networks characteristics suggest that fusion is a mechanism required for the proper respiratory activity and metabolic efficiency of mitochondria, as well as, for the complementation, stabilization, and protection of mitochondrial
DNA (mtDNA) [33, 34]. Actually, the functionality of a damaged mitochondria can be complemented by fusion with a neighboring integral mitochondria and possibly be restored [26]. In addition, the transfer of mtDNA or whole mitochondria between cells can occur in vitro and rescue aerobic respiration in cells without functional mitochondria [35].

Both mitochondrial fission and fusion seem to be required to maintain mitochondrial function. However, the mechanisms differ. Fission occurs probably to protect function by facilitating equal segregation of mitochondria into daughter cells during cell division and to improve distribution of mitochondria along microtubules tracks. In addition, fission may also help to isolate segments of damaged mitochondria, promoting their clearance by macroautophagy as discussed below [36]. Besides to maintain normal mitochondrial functions, mitochondrial fusion, as well as mitochondrial fission have also been associated with cell death/survival mechanisms [37, 38].

Sophisticated genetic and biochemical studies in several organisms, ranging from Drosophila melanogaster and baker's yeast Saccharomyces cerevisiae to mammalian cells, have provided valuable inroads into understanding the biological processes of mitochondrial dynamics and greatly accelerated the identification and characterization of the major components of the fusion and fission machineries and their regulation [39-42]. The critical balance between mitochondrial dynamic systems is greatly maintained by a large group of conserved proteins, the dynamin-related GTPases.

2.2. Mitochondrial Fusion. Mitochondrial fusion involves mechanisms distinct or at least more complex from those that govern membrane fusion in the secretory pathway or other membrane-bound organelles. The fusion process can be divided in at least three events: docking, fusion of the outer membrane, and fusion of the inner membrane. Mitofusins Mfn1 and Mfn2 are engaged in mitochondria tethering and outer membrane fusion. Opal is involved in the outer membrane fusion step with the inner membrane contacts and can have a direct physical contribution to the fusion of inner membranes itself [43]. Moreover, it has been described that mutations in Opal and Mfn2 genes cause Kjer's disease/autosomal dominant optic atrophy and Charcot-Marie Tooth type 2A neuropathy in humans [44].

To date, several mechanisms, such as protein-protein interactions, posttranslational modifications, protein turnover and the lipid environment, have been proposed as regulators of mitochondrial fusion. Indeed, the modulation of the amount of Mfn protein regulates the extent of mitochondrial fusion. In addition, the ability of Mfn1 and 2 to oligomerize and hydrolyze GTP to promote the membrane rearrangements is another point of regulation of mitochondrial fusion [45]. The mechanism of inner membrane fusion is unknown, but findings indicate that two distinct isoforms of Opa1, resulting from proteolytic processing, are necessary for successful fusion events [46, 47].

Two mammalian members of proapoptotic Bcl-2 family members, Bax and Bak, induce mitochondrial fusion by 
regulating the assembly and submitochondrial distribution of Mfn2. Intriguingly, their mitochondrial localization is required for apoptosis induction and for cell survival, pointing to an intimate connection of mitochondrial remodeling and programmed cell death [48].

2.3. Mitochondrial Fission. While the precise mechanism of mitochondrial fission in mammals is largely unknown, most insights concerning this mechanism have come from studies in yeast. It is thought that mitochondrial fission in mammals follows the same steps as in yeast: Drp1 is recruited to mitochondria, and constriction of the membranes takes place via direct or indirect interaction with hFis1 [49].

The major regulatory mechanisms that control mitochondrial fission seem to involve posttranslational modifications of Drp1 which determine its localization, dynamics, and activity. One of the posttranslational modifications that regulate mitochondrial division is ubiquitinylation. Drp1 ubiquitinylation seems to regulate the kinetics of Drp1 binding to the mitochondrial surface. Thus, ubiquitin conjugation might regulate the subcellular trafficking, assembly of Drp1, and influence the rate of mitochondrial division [50]. Mitochondrial fission is also regulated in part by phosphorylation. It was demonstrated that phosphorylation of Drp1 at serine 616 by the cyclin B-dependent kinase (CDK1) induces fragmentation of mitochondria during mitosis [51]. Unlike phosphorylation by CDK1, protein kinase A-(PKA, cAMPdependent protein kinase-) dependent phosphorylation of a different serine residue (S637) can decrease Drp1 GTPase activity [52]. Thus, it seems that phosphorylation of Drp1 by different kinases at different amino acid residues causes opposite effects. Although the initial study indicate that Drp1 phosphorylation can modulate the frequency of mitochondrial division, it remains to be understood if the fission competent phospho-Drp1 (S616) is always phosphorylated by CDK1 or if this mechanism is only active during the cell cycle [39]. Moreover, dephosphorylation of S637 by the $\mathrm{Ca}^{2+}$-dependent phosphatase calcineurin promotes Drp1 translocation to mitochondria and subsequently mitochondrial fission [53].

In addition to frequent cycles of fusion and fission in a "kiss and run" pattern, mitochondria are also turned over during the neuronal lifetime, being replaced throughout organelle biogenesis.

2.4. Mitochondrial Biogenesis. Mitochondria cannot be made de novo. The formation of new mitochondria, called mitochondrial biogenesis, encompasses all processes involved in maintenance and growth of these organelles, as well as the ones required for their division and segregation during the cell cycle. Thus, mitochondrial biogenesis is an extremely complex process that requires the synthesis, import, and incorporation of proteins and lipids to the existing mitochondrial reticulum, as well as the replication of the mtDNA. Although mitochondria have their own DNA, mitochondrial genome encodes only a small but essential group of 13 proteins, including hydrophobic proteins of the electron transport chain, as well as mitochondrial tRNAs and rRNA. The vast majority of the mitochondrial proteins are encoded by nuclear genes. Therefore, mitochondrial biogenesis requires the coordinated transcription of the large number of mitochondrial genes in the nucleus, as well as of the fewer but essential genes in mitochondria. This implies the cooperation of two genomes, which is a potential challenge to the neuronal cell. This could limit mitochondrial biogenesis to the cell body and require that all mitochondrial renewal in the distal axon occurs by axonal transport. However, there is also a reason to believe that mitochondrial biogenesis could occur within the axon. It is now well established that local protein synthesis occurs in axons [54$56]$ and is essential for axonal functions including direction [57-59], regeneration [56, 60-62], and maintenance of mitochondrial membrane polarization [63]. In addition, transcripts for nuclear-encoded mitochondrial proteins are among the species found in axons [64]. Moreover, mitochondria are found in close proximity to translation sites in the axon [65]. Several studies have revealed that components of the mitochondrial replication apparatus are located outside the perinuclear region in nonneuronal cells [66-70] and in SH-SY5Y neuron-like cells [70]. More recently, it was shown that mtDNA replication and mitochondrial fission and fusion occur in the distal axons of peripheral neurons in culture, showing that a portion of mitochondrial biogenesis, like protein biosynthesis, does occur in the axon at significant distances from the cell body [71].

\subsection{The Relevance of Mitochondrial Dynamics for Neuronal} Integrity. Neurons are highly specialized cells that undergo unique challenges in carrying out their important physiological functions. First, neurons are active cells and thus require large amounts of energy. Furthermore, some neurons have extremely long processes, with axons extending up to one meter in motor neurons. Thus, neurons must transmit energy across long distances. They are also long-lived postmitotic and highly interactive cells whose major function is communication. Therefore, neurons rely profoundly on the rapid and versatile distribution of mitochondrial activity and on mitochondrial biogenesis over the time and space [72]. In neurons, the mitochondrial fission/fusion machinery is intimately and critically involved in the formation of synapses and dendritic spines. Actually, both fusion and fission mechanisms contribute for the full-mitochondrial lifecycle and any disruption on their balance could change the steady-state distribution of mitochondrial span. Since mitochondria cannot be made de novo, the fission of preexisting organelles is essential for generation of the new mitochondria. Distressing fission or fusion mechanisms either by inhibiting expression of the fission protein Drp1 [73-75] or by overexpressing the fusion protein Mfn1 [76] has been shown to prevent mitochondria from distributing to synapses, leading to a loss of mitochondria from dendritic spines and, consequently, to a reduction of synapse formation. On the other hand, counteracting this process by overexpressing Drp1 and/or promoting its effects on dendritic mitochondria restores synapse formation [77]. In addition, mitochondrial fusion has been directly implicated in preventing the accumulation of damaged $\mathrm{mtDNA}[28,78]$. More recently, mitochondrial fission has also been shown 
to be critical to mtDNA maintenance [79]. Accordingly, it seems that fission and fusion serve the same purposes along neurons: fission is the final step in mitochondrial duplication, whereas fusion dilutes errors in mtDNA, being both processes required to protect mitochondrial integrity and function $[30,80]$.

Thus, considering how much neurons depend on mitochondria, it should come as no surprise that there is a strong association between mitochondrial dysfunction and neurodegenerative diseases. However, key questions arise: why specific areas of the brain are differentially affected? and/or why only selective groups of neurons die in PD? What makes these specific groups of distinct neurons particularly susceptible to degeneration is not known yet. However, it is now well established that the degeneration of PD affects not only dopaminergic neurons, but many other neurons in the central nervous system, including populations in the brainstem, as well as in the subcortical and cortical regions [81]. Neurons of these areas have common features as they have long and thin axons, which have little or no myelination [82]. Neurons with these features are more vulnerable to degeneration and require high energy demands and so they are particularly dependent on suitable mitochondrial dynamics. In addition, it was recently reported that mitochondrial mass and size are correlated with cell size and that dopaminergic neurons in the SNpc, ventral tegmental area, and interfascicular nucleus have a significantly a smaller area of the cytoplasm occupied by mitochondria than in the neighboring nondopaminergic neurons. This suggests that the low mitochondria mass of the nigral dopaminergic neurons may contribute to their vulnerability to degeneration [83]. Moreover, mitochondrial content in axons, synapses, and dendrites (collectively known as neurites) plays a crucial role in regulating outgrowth and synaptic remodeling into adulthood [77, 84]. Alterations on neurites remodeling and plasticity due to aging and disease processes probably contribute to memory loss and neurodegeneration. In fact, the dynamic remodeling of preexisting mitochondria may play a more prominent role than biogenesis in regulating mitochondrial content in neuritis $[75,85]$.

\section{Novel Implications of Mitochondrial Dynamics in PD Pathogenesis}

\subsection{Mitochondrial Motility: An On-Track Sliding-Dependent} Cellular Process. Proper functioning of mitochondria depends on their intracellular location which is decisively governed by aspects of mitochondrial spatial arrangement and motility beyond fusion and fission. Those aspects are critically important when we look to cell polarity, such as in neurons [72], which require mitochondria at sites distant from the cell body. Transport of mitochondria and their positioning within neurons are microtubule-dependent and, in turn, transport on microtubules depends on the molecular motors kinesins and dyneins [86]. It was demonstrated that the dynein-dynactin motor complex interacts with Drp1 and recruits the protein to the mitochondrial surface [87]. In addition, disruption of F-actin also blocks translocation of Drp1 and, subsequently, mitochondrial fission [88]. Very recently, Milton and Miro were shown to localize to mitochondria in mammalian neurons and interact with each other in vivo in the brain [89, 90]. Miro has also been shown to bind directly to kinesin heavy chain KIF5 in a $\mathrm{Ca}^{2+}$-sensitive manner $[89,90]$ and together with Milton (Miro-Milton complex) allows mitochondria to move along the microtubule network and supports ondemand distribution of mitochondria. In mammalian cells, manipulation of Mirol was shown to dramatically affect mitochondrial morphology and these effects, in the particular case of fission, appear to involve Drp1 activation [91]. In Drosophila, loss of Miro-dependent transport pathway results in depletion of mitochondria in dendrites and axons, inducing neurotransmission defects during prolonged stimulation [92]. Furthermore, defects in both fusion and fission have been shown to impair mitochondrial movement. Apparently, the large interweave of highly connected mitochondria in fission-deficient cells prevents an efficient movement, particularly into thin locations such as neuronal processes $[75,77]$. In fusion-deficient cells, the cause of decreased motility is less obvious. However, Mfndeficient mitochondria display loss of directed movement, travelling in a manner reminiscent of Brownian motion [45]. In neurons lacking mitochondrial fusion, swollen and nucleoid-deficient mitochondria cluster at dendritic junctions and are unable to enter the distal, smaller diameter branches. Thus, both clustering of mitochondria in the cell body and the blockage of efficient entry into neurites may contribute to the lack of mitochondria in the axons and dendrites [37]. Collectively, pieces of evidence lead to the hypothesis that the effect of mitochondrial fusion and fission disturbances could secondarily impair motility and, on the other hand, transport defects affect mitochondrial shape.

3.2. Mitophagy: A Major Line of Defense against Mitochondrial Damage. As mentioned before, perturbations in mitochondrial dynamics, throughout fusion or fission alterations, can impair the energy provision by mitochondria in mammalian neurons. Nevertheless, cells have developed sophisticated systems to deal with the diverse challenges imposed on mitochondrial functional integrity. These systems could comprise a "multistep" mitochondrial quality control network that assists to the spatial segregation of damaged mitochondria. The first tier of quality control is provided by both molecular chaperones and the intracellular proteolytic system, which selectively remove excess and damaged proteins from mitochondria outer membrane. A second step in mitochondrial quality control could be mediated by fusion of damaged mitochondria with neighboring mitochondria [26]. However, severe injury of mitochondria impairs fusion and further activates fission-dependent fragmentation and sequestration by an autophagic process, termed mitophagy. Accumulating data suggests that mitochondrial dysfunction by itself triggers mitophagy [93]. In fact, it was demonstrated that mitochondria-derived ROS, at low concentrations, may act as signaling molecules and trigger mitophagy throughout redox regulation of Atg4, an essential cysteine protease in the autophagic pathway [94]. Similarly, Gomes and 
Scorrano also provided evidence that the profission mitochondrial protein Fis1 induces mitochondrial fragmentation and enhances mitophagy. Nevertheless, these changes were correlated with mitochondrial dysfunction rather than with fragmentation [95]. As well, mitochondrial fission was also shown to be an important step for the autophagic clearance of depolarized or damaged mitochondria, since overexpression of Drp1 promotes mitophagy [36].

Additional studies showed the involvement of Parkin, PINK, and LRRK2 in the regulation of mitochondrial clearance and homeostasis. Parkin was found to be selectively recruited to dysfunctional mitochondria with low membrane potential in mammalian cells targeting mitochondria towards the autophagic-lysosomal pathway [96]. Moreover, translocation of Parkin to mitochondria is voltage dependent and does not depend on changes in pH or ATP levels [97]. Thus, these findings suggest that Parkin may act as a sensor for mitochondrial integrity and limit mitochondrial damage by acting in a pathway that identifies and eliminates damaged mitochondria from the mitochondrial network.

PINK1 and Parkin can cooperate in a common pathway that is involved in the protection of mitochondrial integrity and function. Indeed, it was reported that cooverexpression of Parkin and PINK1 collapses the normal tubular mitochondrial network into mitochondrial aggregates and/or large perinuclear clusters, many of which are associated to LC3-enriched autophagic vacuoles [98, 99]. These results suggest that both proteins are involved in the modulation of mitochondrial trafficking, especially to the perinuclear region, a subcellular area associated with autophagy-lysosomal degradation [98]. Moreover, PINK1 accumulation on mitochondria is both necessary and sufficient for Parkin recruitment to mitochondria [98, 100]. Recent studies have shown that PINK1 accumulates selectively on dysfunctional mitochondria and its kinase activity together with its mitochondrial sequence is a prerequisite to induce translocation of Parkin to depolarized mitochondria. Subsequently, Parkin mediates the poly-ubiquitylation of VDAC-1 (voltage-dependent anion channel 1) [101], Mfn1, and Mfn2 among other mitochondrial proteins [102]. Additionally, the autophagic adaptor p62/SQSTM1 is recruited to mitochondrial clusters and is essential for the clearance of mitochondria by mitophagy [101]. Taken together, these data provide a functional link between Parkin, PINK1 and mitophagy which is implicated in the pathogenesis of PD.

Moreover, the role of LRRK2 in regulating autophagy was also addressed. Interestingly, it was shown that LRRK2 specifically localizes to specific membrane subdomains and endosomal-autophagic structures, suggesting a functional relationship between LRRK2 and mitophagy [103]. Moreover, increased autophagic activity upon LRRK2 knockdown was observed, which indicates that LRRK2 may normally act as a negative regulator of autophagy [103]. Alternatively, LRRK2 regulation in neurite blunting and remodeling requires autophagy [104]. Thus, by impairing this pathway, mutations in Parkin, PINK1, and LRRK2 may alter autophagy-dependent mitochondrial turnover which, in turn, may cause the accumulation of defective mitochondria and, ultimately, neurodegeneration in PD. Conversely,
Beclin 1-independent autophagy/mitophagy contributes to cell death elicited by the PD toxins $\mathrm{MPP}^{+}$and 6-OHDA [105], causing neurite retraction in cells expressing the G2019S PD-linked mutation in LRRK2 [105]. However, excessive or incomplete autophagy without suitable regenerative biogenesis, due to deficient retrograde trafficking of vesicles can lead to "autophagic stress" [106], which may ultimately lead to neuronal degeneration.

3.3. Failure of an Exquisite Network of Quality Control in PD?. An early study by de Mattos and coworkers [107] in brains from PD patients demonstrated mitochondrial tumefaction and deposits of amorphous substance into mitochondria and axons distinctively absent in the control patients. Consistent findings were further found by Trimmer and colleagues [108] in PD cybrids and in studies with the same model in our laboratory. Mitochondria in PD cybrid cells were found enlarged or swollen with disrupted cristae and a discontinuous outer membrane [16]. In contrast, sublethal concentrations of $\mathrm{MPP}^{+}$and rotenone, two mitochondrial complex I inhibitors that induce parkinsonian syndrome in vivo, were shown to promote Drp1-dependent mitochondrial fragmentation [109], to decrease mitochondrial mobility $[110,111]$ and to disrupt microtubule dynamics [112-114]. However, chronic low-dose exposure to rotenone and $\mathrm{MPP}^{+}$was shown to induce mitochondria swelling and decreased anterograde transport of mitochondria and vesicles, probably due to a reduction of ATP supply to molecular motors [110, 115].

Additional studies of mammalian PINK and parkin models have been suggestive of their involvement on the regulation of mitochondrial turnover, dynamics, and cellular homeostasis. The role for parkin was revealed using a Drosophila parkin null mutant or by overexpression of one pathogenic parkin mutation, showing severe mitochondrial pathology, reduced lifespan, and increased apoptosis [116, 117]. It was also found that functional parkin is necessary for proper mitochondrial organization and morphology throughout spermatid development in Drosophila [118]. Additionally, mitochondrial respiratory defects and morphological abnormalities have been reported in brains of parkinknockout, parkin-mutant transgenic mice $[119,120]$, and in leukocytes from PD patients with parkin mutations [121, 122]. Primary fibroblasts from patients carrying mutations in parkin, or control fibroblasts treated with siRNA against parkin, revealed lower mitochondrial membrane potential, lower ATP levels, and increased susceptibility to rotenone toxicity [121]. The fibroblasts also exhibited mitochondrial morphological abnormalities, exhibiting mitochondria that were longer and more highly branched. Interestingly, there was a relationship between those mitochondrial deficits and increased length and branching, as well as between reduced complex I activity and mitochondrial branching. Thus, as parkin is not specifically located into mitochondria and must be translocated to this organelle, it is conceivable that its effects on mitochondrial morphology are tied into a larger pathway that mediates mitochondrial maintenance. Actually, within mitochondria, parkin mainly localizes in the inner membrane and matrix, where it has been shown 


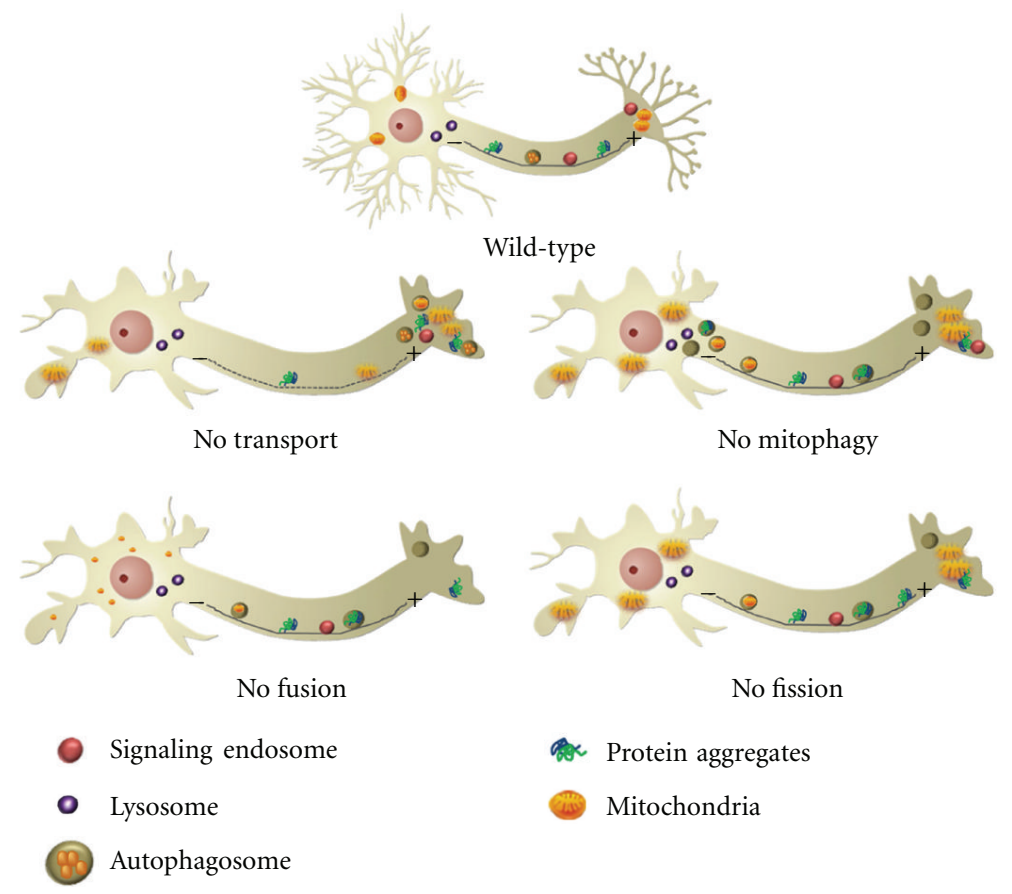

FIgURE 1: Potential susceptibility of neurons to mitochondrial dysfunction and impaired mitochondrial turnover. (Wild type) In healthy neurons, mitochondria, prosurvival signals associated to signaling endosomes, and autophagomes enclosing damaged organelles or protein aggregates are able to travel long distances from the cell periphery to perinuclear region in the cell body, where most lysosomes are concentrated. (No transport) Disruption of microtubule network and subsequent defects on retrograde transport prevent the proper distribution of mitochondria and the efficient transport of autophagy substrates towards lysosomes for degradation, which can lead to defects in energy supply and cargos clearance by autophagy. (No mitophagy) Blockage of autophagic activity seems to be responsible for the accumulation of damaged mitochondria, toxic protein products, aggregates, and leaking autophagic vesicles, all of which have a negative effect on neuronal functioning and survival, precipitating the "dying-back"-type of axonal degeneration. (No fusion) The absence of mitochondrial fusion may result in an accumulation of damaged mitochondria or decreased healthy mitochondria at the nerve terminal. Mitochondria secondarily have defects in motility that prevent proper distribution within the axon and in the periphery. (No fission) In the absence of mitochondrial fission, most of the mitochondrial population is extensively long and interconnected, and a subset shows ultrastructural defects and dysfunction. The large mitochondria clusters within dendrites are not efficiently transported and/or engulfed by autophagosomes towards cell body for lysosomal degradation. (MT: microtubule tracks oriented along the axon with plus (+) ends distal and $(-)$ ends proximal to the cell body).

to enhance transcription and replication of mtDNA and to induce mitochondrial proliferation [123]. This effect seems to be mediated by the interaction of parkin with mitochondrial transcription factor A (TFAM), a protein that regulates mtDNA transcription by directly binding and coating mtDNA [123]. In PC12 cells differentiated into neurons by nerve growth factor, parkin was located in the outer mitochondrial membrane, where prevented ceramideinduced mitochondrial swelling, cytochrome c release, caspase activation, and apoptotic cell death [124]. This effect may be related to the above-mentioned ability of parkin to regulate mitochondrial morphology. Intriguingly, complex I inhibition with rotenone seems to induce the release of mitochondrial parkin to the cytosol [123]. In light of these results, complex I deficiency or loss-of-function mutations in parkin may impair the mitochondrial localization of parkin and thus abolish the boosting and beneficial effects of this protein at the mitochondria. Indeed, the protective effect of Parkin is abolished by PD-causing parkin mutations and proteasome inhibitors, indicating that it is mediated by its $\mathrm{E} 3$ ubiquitin ligase activity [124].
Regarding PINK, a significant decrease in mitochondrial respiration in the striatum of PINK1 knockout mice [125] was observed. Several groups also described mitochondrial abnormalities in pink Drosophila mutants, similar to those of parkin mutant Drosophila [126-128]. Moreover, the protective effect of PINK1 has also been confirmed in primary neuronal cultures and in an in vivo mouse model of PD [129]. In this study, PINK1 suppression by small interfering RNA in primary cortical neurons treated with $\mathrm{MPP}^{+}$promoted neuronal death, while overexpression of wild-type PINK1 protected these neurons against $\mathrm{MPP}^{+}$ toxicity [129]. The protective effect of PINK1 was confirmed as being mediated by its kinase domain and was abolished in PD-related PINK1 mutants [129]. Adenoviral-mediated expression of PINK1 in mice SNpc protected dopaminergic neurons from MPTP-induced cell death, an effect that was abolished by expression of kinase-inactive PINK1 mutants [129]. Surprisingly, the mitochondrial targeting sequence and the mitochondrial localization of PINK1 were not necessary for its protective effect, either in vitro or in vivo, as demonstrated in a mutant PINK1 with impaired 


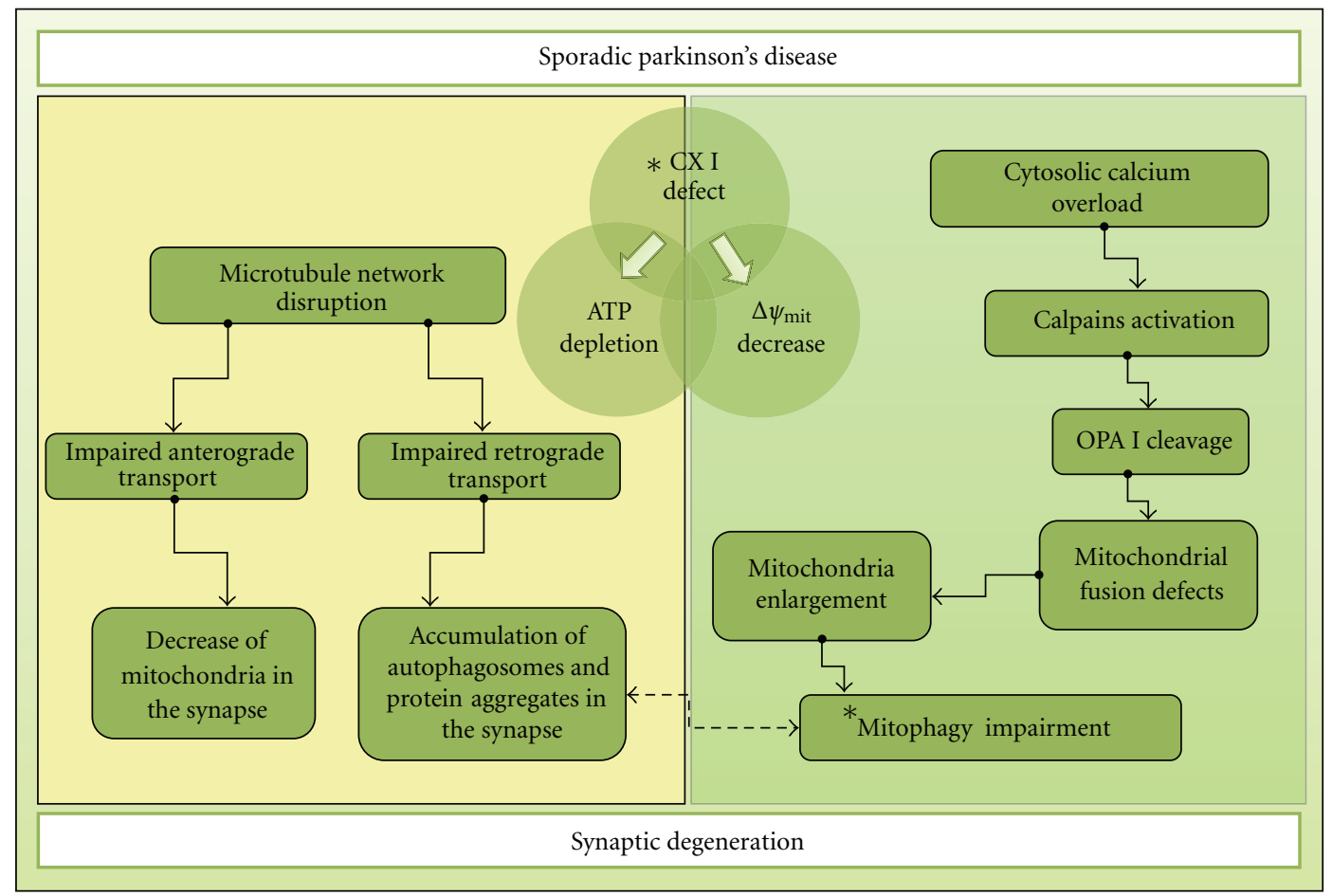

FIGURE 2: Rationale for the contribution of mitochondrial dysfunction to synaptic degeneration in sporadic PD. Mitochondrial dysfunction induced by a complex I defect leads to alterations in mitochondria-dependent metabolism (reduced ATP levels and decreased mitochondrial inner membrane potential). This bioenergetic failure seems to potentiate microtubule network breakdown. Subsequently, when the dynamics and functional integrity of microtubules are compromised, changes in anterograde and retrograde flux along the axon can be impaired. Cargos that are actively transported along the axon include mitochondria, autophagosomes, and proteins. Moreover, a decrease in mitochondrial membrane potential deregulates calcium homeostasis, which leads to the overactivation of calpains. Calpains are key regulators of mitochondrial fusion, since they impair Opal proper function. Alterations in fusion/fission events promote mitochondrial enlargement, which can impair their removal by mitophagy. Indeed, the accumulation of protein aggregates, autophagosomes, and enlarged deficient mitochondria in presynaptic termini is observed at early stages of PD. Our hypothesis implies that mitochondrial metabolism impairment could be responsible for synaptic degeneration in PD. (*Indicates that mutated or overexpressed $\alpha$-synuclein could induce mitochondrial dysfunction and that loss-of-function of Parkin or/and PINK1 can deregulate mitochondrial mitophagy.)

mitochondrial localization [129]. These results indicate that cytoplasmic, rather than mitochondrial, kinase activity of PINK1 is critical for its protective effect. To reconcile these results with the known mitochondrial localization of PINK1, it has been reported that while PINK1 spans the outer mitochondrial membrane, with the $\mathrm{N}$-terminal end inside the mitochondria, the C-terminal kinase domain of PINK1 actually faces the cytosol [130]. Very recently, it was also suggested that PINK1 may also be involved in mitochondrial transport. Weihofen and colleagues demonstrated that Miro and Milton overexpression reverted mitochondria pathology induced by the loss of functional PINK1 [131]. Moreover, PD-associated PINK1 mutations also compromise the selective degradation of depolarized mitochondria maybe due to a decreased physical binding of PINK1 to Parkin. In addition to an impaired PINK1 kinase activity, reduced binding of PINK1 to Parkin leads to failure in mitochondrial clearance, resulting in the accumulation of damaged mitochondria [132].

Both Drosophila and mammalian cells studies show that loss of PINK result in abnormalities in mitochondrial morphology, involving mitochondrial swelling accompanied by decreased or disorganized cristae $[125,133]$, which are remarkably similar to mitochondria in sporadic PD cybrid cell lines [16, 108, 134]. Interestingly, PINK and Parkin flies were shown to have comparable phenotypes. While parkin overexpression reverses the effects of PINK loss-offunction on mitochondrial morphology, the opposite was not observed, suggesting that parkin acts downstream of PINK [126, 128].

However, studies in PD patients fibroblasts carrying PINK mutations and in human HeLa, M17, and SH-SY5Y PINK knockdown cells showed an increase in fragmented mitochondria $[135,136]$. These results may be correlated with the role of PINK in preventing mitochondrial oxidative stress and fission events. Indeed, an increase in mitochondrial fragmentation was reversed by overexpression of Parkin in HeLa and SH-SY5Y cells, which indicates a clearance of the damaged mitochondria.

Foremost, silencing PINK expression in dopaminergic SH-SY5Y cells resulted in progressive loss of mitochondrial function characterized by decreased mtDNA levels, impaired oxidative phosphorylation, and oxidative stress [137]. Nevertheless, in this study the decreases in mtDNA 
and oxidative phosphorylation do not appear to be directly related to Parkin loss of function. Instead, loss of mtDNA can be suggested as the most probable cause of mitochondrial respiratory chain inhibition and consequent oxidative stress. This supports the notion that the combination of both mitochondrial dysfunction and perturbed PINK1 activity increases the susceptibility to oxidative stress or apoptosis as has been reported in brain cells $[125,138]$. Besides, PINK knockdown cells show decreased phosphorylation of Drp1 at S637 through activation of calcineurin phosphatase activity [136]. While PINK overexpressing cells show Drp1 2Dgel mobility consistent with higher phosphorylation states [139]. Because Drp1 phosphorylation at S637 inhibits its ability to mediate fission [52], these results point to a role of PINK as a fission suppressor.

Even though PINK1/Parkin may regulate mitochondrial dynamics in some of the familial PD forms, it seems evident that mitochondrial physiology is the major determinant of mitochondria morphology in sporadic cases. Particularly, it is believed that the structural composition of mitochondria may determine their propensity for fragmentation and self-elimination, and that this may be influenced by the metabolic status within the cell. Accordingly, an RNAi screening for mitochondrial proteins in Caenorhabditis elegans demonstrated that the knockdown of more than $80 \%$ of mitochondrial genes lead to mitochondrial fragmentations and/or aggregation, showing that mitochondria morphology maintenance requires a huge number of proteins and not necessarily the specific action of mitochondrial fusion or fission machinery [140].

Furthermore, ultrastructural examination indeed revealed "autophagic stress" in melanized neurons of the SNpc in PD patients [141]. Moreover, accumulated autophagosomes have been observed in human PD nigral neurons [141], but not in nigral neurons during normal aging. Alterations in macroautophagy are also implicated in PD since its inhibition leads to $w t \alpha$-synuclein accumulation, suggesting that this lysosomal pathway is also involved in normal $\alpha$-synuclein turnover [142]. Very recently, it was also demonstrated that $\alpha$-synuclein overexpression impairs macroautophagy in mammalian cells and in transgenic mice [143]. Interestingly, recent work in our laboratory has shown that cells with an mtDNA-mediated mitochondrial dysfunction have enhanced formation autophagic vacuoles but present decreased degradation ability, suggesting that the autophagic clearance is impaired in PD. We have also observed that macroautophagy inhibition increased $\alpha$-synuclein oligomerization and prompts apoptosis (DM Arduíno, unpublished data).

\section{Concluding Remarks and Perspectives}

There is a growing body of evidence supporting that alterations in mitochondrial dynamics are implied in PD pathogenesis (Figure 1). However, there are still some controversies among the different models of disease, namely, concerning the mitochondrial morphology changes and the mechanisms that control mitochondrial shape and function. Common approaches, such as those modulating fusion/fission, are scarce to explain how mitochondrial abnormalities occur in certain disease states. Undoubtedly, it seems evident that cellular physiology is the major determinant of mitochondria function and morphology. In addition, mitochondrial fusion and fission are not isolated in the cell and other intrinsic cellular alterations, such as impaired vesicular trafficking and axonal transport, intracellular degradation systems and mitochondrial metabolism, have also been described in PD and could further alter mitochondrial biogenesis, turnover, and maintenance.

Taken in account the available data, we propose the mitochondrial cascade hypothesis to explain PD pathogenesis. Mitochondrial dysfunction induced by a complex I defect leads to alterations in mitochondrial-dependent metabolism (reduced ATP levels and decrease in mitochondrial membrane potential) [144]. This bioenergetic failure seems to play a role in microtubule network breakdown [145]. Subsequently, when microtubules dynamic and functional integrity are compromised, changes in anterograde and retrograde flux along the axon can lead to defects in the supply and clearance of mitochondria [146]. Moreover, a decrease in mitochondrial membrane potential potentiates an increase in cytosolic calcium which leads to calpains overactivation [147]. In addition, mitochondria with low inner membrane potential have a decrease in Opal levels and do not fuse [148]. Accordingly, calpain activation seems to mediate Opa1 loss of function [149]. Together, these findings point to an accumulation of enlarged mitochondria [16], that will impair efficient removal by autophagy. Our hypothesis implies that mitochondrial failure may be the initial event in sporadic PD, although it has a prominent role in some of PD familial forms (Figure 2).

Understanding this complex molecular network and indentifying the factors that control all of these interrelated mechanisms is currently a priority and a challenge for future studies.

\section{Acknowledgments}

Work in our laboratory is supported by funds from PTDC/ SAU-NEU/102710/2008. D. M. Arduíno and A. R. Esteves are also supported by Ph.D. Fellowships (SFRHD/BD/38743/ 2007 and SFRH/BD/32470/2006, resp.) from Foundation for Science and Technology (FCT-MCTES, Portugal).

\section{References}

[1] L. S. Forno, "Neuropathology of Parkinson's disease," Journal of Neuropathology and Experimental Neurology, vol. 55, no. 3, pp. 259-272, 1996.

[2] R. Banerjee, A. A. Starkov, M. F. Beal, and B. Thomas, "Mitochondrial dysfunction in the limelight of Parkinson's disease pathogenesis," Biochimica et Biophysica Acta, vol. 1792, no. 7, pp. 651-663, 2009.

[3] M. F. Beal, "Mitochondria, oxidative damage, and inflammation in Parkinson's disease," Annals of the New York Academy of Sciences, vol. 991, pp. 120-131, 2003. 
[4] A. H. Schapira, "Mitochondria in the aetiology and pathogenesis of Parkinson's disease," The Lancet Neurology, vol. 7, no. 1, pp. 97-109, 2008.

[5] S. M. Cardoso, A. R. Esteves, D. M. Arduíno, A. F. Domingues, and C. R. Oliveira, "The crucial role of mitochondria in Parkinson's disease," Recent Research Developments in Neuroscience, vol. 3, pp. 43-84, 2009.

[6] D. M. Arduíno, A. R. Esteves, C. R. Oliveira, and S. M. Cardoso, "Mitochondrial metabolism modulation: a new therapeutic approach for Parkinson's disease," CNS and Neurological Disorders Drug Targets, vol. 9, no. 1, pp. 105119,2010

[7] J. W. Langston, P. Ballard, J. W. Tetrud, and I. Irwin, "Chronic parkinsonism in humans due to a product of meperidineanalog synthesis," Science, vol. 219, no. 4587, pp. 979-980, 1983.

[8] R. S. Burns, P. A. LeWitt, and M. H. Ebert, "The clinical syndrome of striatal dopamine deficiency. Parkinsonism induced by 1-methyl-4-phenyl-1,2,3,6-tetrahydropyridine (MPTP)," New England Journal of Medicine, vol. 312, no. 22, pp. 1418-1421, 1985.

[9] A. H. V. Schapira, J. M. Cooper, D. Dexter, P. Jenner, J. B. Clark, and C. D. Marsden, "Mitochondrial complex I deficiency in Parkinson's disease," Lancet, vol. 1, no. 8649, p. 1269, 1989.

[10] W. D. Parker, S. J. Boyson, and J. K. Parks, "Abnormalities of the electron transport chain in idiopathic Parkinson's disease," Annals of Neurology, vol. 26, no. 6, pp. 719-723, 1989.

[11] N. Barroso, Y. Campos, R. Huertas et al., "Respiratory chain enzyme activities in lymphocytes from untreated patients with Parkinson disease," Clinical Chemistry, vol. 39, no. 4, pp. 667-669, 1993.

[12] H. Yoshino, Y. Nakagawa-Hattori, T. Kondo, and Y. Mizuno, "Mitochondrial complex I and II activities of lymphocytes and platelets in Parkinson's disease," Journal of Neural Transmission Parkinson's Disease and Dementia Section, vol. 4, no. 1, pp. 27-34, 1992.

[13] S. M. Cardoso, P. I. Moreira, P. Agostinho, C. Pereira, and C. R. Oliveira, "Neurodegenerative pathways in Parkinson's disease: therapeutic strategies," Current Drug Targets CNS and Neurological Disorders, vol. 4, no. 4, pp. 405-419, 2005.

[14] R. H. Swerdlow, J. K. Parks, S. W. Miller et al., "Origin and functional consequences of the complex I defect in Parkinson's disease," Annals of Neurology, vol. 40, no. 4, pp. 663-671, 1996.

[15] D. S. Cassarino, C. P. Fall, R. H. Swerdlow et al., "Elevated reactive oxygen species and antioxidant enzyme activities in animal and cellular models of Parkinson's disease," Biochimica et Biophysica Acta, vol. 1362, no. 1, pp. 77-86, 1997.

[16] A. R. F. Esteves, A. F. Domingues, I. L. Ferreira et al., "Mitochondrial function in Parkinson's disease cybrids containing an nt2 neuron-like nuclear background," Mitochondrion, vol. 8, no. 3, pp. 219-228, 2008.

[17] A. R. Esteves, J. Lu, M. Rodova et al., "Mitochondrial respiration and respiration-associated proteins in cell lines created through Parkinson's subject mitochondrial transfer," Journal of Neurochemistry, vol. 113, no. 3, pp. 674-682, 2010.

[18] J. Q. Kwong, M. F. Beal, and G. Manfredi, "The role of mitochondria in inherited neurodegenerative diseases," Journal of Neurochemistry, vol. 97, no. 6, pp. 1659-1675, 2006.
[19] B. Thomas and M. F. Beal, "Parkinson's disease," Human Molecular Genetics, vol. 16, no. 2, pp. R183-R194, 2007.

[20] J. M. Jones, P. Datta, S. M. Srinivasula et al., "Loss of Omi mitochondrial protease activity causes the neuromuscular disorder of mnd2 mutant mice," Nature, vol. 425, no. 6959, pp. 721-727, 2003.

[21] S. Biskup, D. J. Moore, F. Celsi et al., "Localization of LRRK2 to membranous and vesicular structures in mammalian brain," Annals of Neurology, vol. 60, no. 5, pp. 557-569, 2006.

[22] S. Saha, M. D. Guillily, A. Ferree et al., "LRRK2 modulates vulnerability to mitochondrial dysfunction in Caenorhabditis elegans," Journal of Neuroscience, vol. 29, no. 29, pp. 9210 9218, 2009.

[23] J. Jin, C. Hulette, Y. Wang et al., "Proteomic identification of a stress protein, mortalin/mthsp70/GRP75: relevance to Parkinson disease," Molecular and Cellular Proteomics, vol. 5, no. 7, pp. 1193-1204, 2006.

[24] M. Shi, J. Bradner, T. K. Bammler et al., "Identification of glutathione S-transferase pi as a protein involved in Parkinson disease progression," American Journal of Pathology, vol. 175, no. 1, pp. 54-65, 2009.

[25] J. Bereiter-Hahn, M. Vöth, S. Mai, and M. Jendrach, "Structural implications of mitochondrial dynamics," Biotechnology Journal, vol. 3, no. 6, pp. 765-780, 2008.

[26] S. A. Detmer and D. C. Chan, "Functions and dysfunctions of mitochondrial dynamics," Nature Reviews Molecular Cell Biology, vol. 8, no. 11, pp. 870-879, 2007.

[27] A. B. Knott, G. Perkins, R. Schwarzenbacher, and E. BossyWetzel, "Mitochondrial fragmentation in neurodegeneration," Nature Reviews Neuroscience, vol. 9, no. 7, pp. 505-518, 2008.

[28] K. Nakada, K. Inoue, T. Ono et al., "Inter-mitochondrial complementation: mitochondria-specific system preventing mice from expression of disease phenotypes by mutant mtDNA," Nature Medicine, vol. 7, no. 8, pp. 934-940, 2001.

[29] S. I. Arimura, J. Yamamoto, G. P. Aida, M. Nakazono, and N. Tsutsumi, "Frequent fusion and fission of plant mitochondria with unequal nucleoid distribution," Proceedings of the National Academy of Sciences of the United States of America, vol. 101, no. 20, pp. 7805-7808, 2004.

[30] H. Chen, A. Chomyn, and D. C. Chan, "Disruption of fusion results in mitochondrial heterogeneity and dysfunction," Journal of Biological Chemistry, vol. 280, no. 28, pp. 2618526192, 2005.

[31] V. P. Skulachev, "Mitochondrial filaments and clusters as intracellular power-transmitting cables," Trends in Biochemical Sciences, vol. 26, no. 1, pp. 23-29, 2001.

[32] G. Twig, S. A. Graf, J. D. Wikstrom et al., "Tagging and tracking individual networks within a complex mitochondrial web with photoactivatable GFP," American Journal of Physiology, vol. 291, no. 1, pp. C176-C184, 2006.

[33] D. C. Chan, "Mitochondria: dynamic organelles in disease, aging, and development," Cell, vol. 125, no. 7, pp. 1241-1252, 2006.

[34] B. Westermann, "Merging mitochondria matters: cellular role and molecular machinery of mitochondrial fusion," EMBO Reports, vol. 3, no. 6, pp. 527-531, 2002.

[35] J. L. Spees, S. D. Olson, M. J. Whitney, and D. J. Prockop, "Mitochondrial transfer between cells can rescue aerobic respiration," Proceedings of the National Academy of Sciences of the United States of America, vol. 103, no. 5, pp. 1283-1288, 2006. 
[36] G. Twig, A. Elorza, A. J. A. Molina et al., "Fission and selective fusion govern mitochondrial segregation and elimination by autophagy," EMBO Journal, vol. 27, no. 2, pp. 433-446, 2008.

[37] H. Chen, J. M. McCaffery, and D. C. Chan, "Mitochondrial fusion protects against neurodegeneration in the cerebellum," Cell, vol. 130, no. 3, pp. 548-562, 2007.

[38] D. F. Suen, K. L. Norris, and R. J. Youle, "Mitochondrial dynamics and apoptosis," Genes and Development, vol. 22, no. 12, pp. 1577-1590, 2008

[39] K. L. Cerveny, Y. Tamura, Z. Zhang, R. E. Jensen, and H. Sesaki, "Regulation of mitochondrial fusion and division," Trends in Cell Biology, vol. 17, no. 11, pp. 563-569, 2007.

[40] E. M. Coonrod, M. A. Karren, and J. M. Shaw, "Ugolp is a multipass transmembrane protein with a single carrier domain required for mitochondrial fusion," Traffic, vol. 8, no. 5, pp. 500-511, 2007.

[41] S. Martens and H. T. McMahon, "Mechanisms of membrane fusion: disparate players and common principles," Nature Reviews Molecular Cell Biology, vol. 9, no. 7, pp. 543-556, 2008.

[42] B. Westermann, "Molecular machinery of mitochondrial fusion and fission," Journal of Biological Chemistry, vol. 283, no. 20, pp. 13501-13505, 2008.

[43] D. C. Chan, "Mitochondrial fusion and fission in mammals," Annual Review of Cell and Developmental Biology, vol. 22, pp. 79-99, 2006.

[44] M. Liesa, M. Palacín, and A. Zorzano, "Mitochondrial dynamics in mammalian health and disease," Physiological Reviews, vol. 89, no. 3, pp. 799-845, 2009.

[45] H. Chen, S. A. Detmer, A. J. Ewald, E. E. Griffin, S. E. Fraser, and D. C. Chan, "Mitofusins Mfn1 and Mfn2 coordinately regulate mitochondrial fusion and are essential for embryonic development," Journal of Cell Biology, vol. 160, no. 2, pp. 189-200, 2003.

[46] L. Griparic, T. Kanazawa, and A. M. Van Der Bliek, "Regulation of the mitochondrial dynamin-like protein Opal by proteolytic cleavage," Journal of Cell Biology, vol. 178, no. 5, pp. 757-764, 2007.

[47] Z. Song, H. Chen, M. Fiket, C. Alexander, and D. C. Chan, "OPA1 processing controls mitochondrial fusion and is regulated by mRNA splicing, membrane potential, and Yme1L," Journal of Cell Biology, vol. 178, no. 5, pp. 749-755, 2007.

[48] M. Karbowski, K. L. Norris, M. M. Cleland, S. Y. Jeong, and R. J. Youle, "Role of Bax and Bak in mitochondrial morphogenesis," Nature, vol. 443, no. 7112, pp. 658-662, 2006.

[49] Y. Yoon, E. W. Krueger, B. J. Oswald, and M. A. McNiven, "The mitochondrial protein hFis1 regulates mitochondrial fission in mammalian cells through an interaction with the dynamin-like protein DLP1," Molecular and Cellular Biology, vol. 23, no. 15, pp. 5409-5420, 2003.

[50] M. Karbowski, A. Neutzner, and R. J. Youle, "The mitochondrial E3 ubiquitin ligase MARCH5 is required for Drp1 dependent mitochondrial division," Journal of Cell Biology, vol. 178, no. 1, pp. 71-84, 2007.

[51] N. Taguchi, N. Ishihara, A. Jofuku, T. Oka, and K. Mihara, "Mitotic phosphorylation of dynamin-related GTPase Drp1 participates in mitochondrial fission," Journal of Biological Chemistry, vol. 282, no. 15, pp. 11521-11529, 2007.

[52] C. R. Chang and C. Blackstone, "Cyclic AMP-dependent protein kinase phosphorylation of Drp1 regulates its GTPase activity and mitochondrial morphology," Journal of Biological Chemistry, vol. 282, no. 30, pp. 21583-21587, 2007.
[53] G. M. Cereghetti, A. Stangherlin, O. Martins De Brito et al., "Dephosphorylation by calcineurin regulates translocation of Drp1 to mitochondria," Proceedings of the National Academy of Sciences of the United States of America, vol. 105, no. 41, pp. 15803-15808, 2008.

[54] E. Koenig and A. Giuditta, "Protein-synthesizing machinery in the axon compartment," Neuroscience, vol. 89, no. 1, pp. $5-15,1999$.

[55] J. Alvarez, A. Giuditta, and E. Koenig, "Protein synthesis in axons and terminals: significance for maintenance, plasticity and regulation of phenotypeWith a critique of slow transport theory," Progress in Neurobiology, vol. 62, no. 1, pp. 1-62, 2000.

[56] J. L. Twiss and J. Van Minnen, "New insights into neuronal regeneration: the role of axonal protein synthesis in pathfinding and axonal extension," Journal of Neurotrauma, vol. 23, no. 3-4, pp. 295-308, 2006.

[57] D. S. Campbell and C. E. Holt, "Chemotropic responses of retinal growth cones mediated by rapid local protein synthesis and degradation," Neuron, vol. 32, no. 6, pp. 10131026, 2001.

[58] G. L. Ming, S. T. Wong, J. Henley et al., "Adaptation in the chemotactic guidance of nerve growth cones," Nature, vol. 417, no. 6887, pp. 411-418, 2002.

[59] K. M. Leung, F. P. Van Horck, A. C. Lin, R. Allison, N. Standart, and C. E. Holt, "Asymmetrical $\beta$-actin mRNA translation in growth cones mediates attractive turning to netrin-1," Nature Neuroscience, vol. 9, no. 10, pp. 1247-1256, 2006.

[60] E. Koenig, "Evaluation of local synthesis of axonal proteins in the goldfish mauthner cell axon and axons of dorsal and ventral roots of the rat in vitro," Molecular and Cellular Neuroscience, vol. 2, no. 5, pp. 384-394, 1991.

[61] J. Q. Zheng, T. K. Kelly, B. Chang et al., "A functional role for intra-axonal protein synthesis during axonal regeneration from adult sensory neurons," Journal of Neuroscience, vol. 21, no. 23, pp. 9291-9303, 2001.

[62] P. Verma, S. Chierzi, A. M. Codd et al., "Axonal protein synthesis and degradation are necessary for efficient growth cone regeneration," Journal of Neuroscience, vol. 25, no. 2, pp. 331-342, 2005.

[63] M. Hillefors, A. E. Gioio, M. G. Mameza, and B. B. Kaplan, "Axon viability and mitochondrial function are dependent on local protein synthesis in sympathetic neurons," Cellular and Molecular Neurobiology, vol. 27, no. 6, pp. 701-716, 2007.

[64] A. E. Gioio, M. Eyman, H. Zhang, Z. S. Lavina, A. Giuditta, and B. B. Kaplan, "Local synthesis of nuclear-encoded mitochondrial proteins in the presynaptic nerve terminal," Journal of Neuroscience Research, vol. 64, no. 5, pp. 447-453, 2001.

[65] R. Martin, B. Vaida, R. Bleher, M. Crispino, and A. Giuditta, "Protein synthesizing units in presynaptic and postsynaptic domains of squid neurons," Journal of Cell Science, vol. 111, no. 21, pp. 3157-3166, 1998.

[66] N. G. Larsson, A. Oldfors, E. Holme, and D. A. Clayton, "Low levels of mitochondrial transcription factor A in mitochondrial DNA depletion," Biochemical and Biophysical Research Communications, vol. 200, no. 3, pp. 1374-1381, 1994.

[67] V. Tiranti, A. Savoia, F. Forti et al., "Identification of the gene encoding the human mitochondrial RNA polymerase (h-mtRPOL) by cyberscreening of the Expressed Sequence Tags database," Human Molecular Genetics, vol. 6, no. 4, pp. 615-625, 1997. 
[68] J. N. Spelbrink, F. Y. Li, V. Tiranti et al., "Human mitochondrial DNA deletions associated with mutations in the gene encoding Twinkle, a phage T7 gene 4-like protein localized in mitochondria," Nature Genetics, vol. 28, no. 3, pp. 223-231, 2001.

[69] M. Falkenberg, M. Gaspari, A. Rantanen, A. Trifunovic, N. G. Larsson, and C. M. Gustafsson, "Mitochondrial transcription factors B1 and B2 activate transcription of human mtDNA," Nature Genetics, vol. 31, no. 3, pp. 289-294, 2002.

[70] J. Magnusson, M. Orth, P. Lestienne, and J. W. Taanman, "Replication of mitochondrial DNA occurs throughout the mitochondria of cultured human cells," Experimental Cell Research, vol. 289, no. 1, pp. 133-142, 2003.

[71] M. Amiri and P. J. Hollenbeck, "Mitochondrial biogenesis in the axons of vertebrate peripheral neurons," Developmental Neurobiology, vol. 68, no. 11, pp. 1348-1361, 2008.

[72] P. J. Hollenbeck and W. M. Saxton, "The axonal transport of mitochondria," Journal of Cell Science, vol. 118, no. 23, pp. 5411-5419, 2005.

[73] D. Tondera, F. Czauderna, K. Paulick, R. Schwarzer, J. Kaufmann, and A. Santel, "The mitochondrial protein MTP18 contributes to mitochondrial fission in mammalian cells," Journal of Cell Science, vol. 118, no. 14, pp. 3049-3059, 2005.

[74] S. Hoppins, L. Lackner, and J. Nunnari, "The machines that divide and fuse mitochondria," Annual Review of Biochemistry, vol. 76, pp. 751-780, 2007.

[75] P. Verstreken, C. V. Ly, K. J. T. Venken, T. W. Koh, Y. Zhou, and H. J. Bellen, "Synaptic mitochondria are critical for mobilization of reserve pool vesicles at Drosophila neuromuscular junctions," Neuron, vol. 47, no. 3, pp. 365378, 2005.

[76] A. Santetl, S. Frank, B. Gaume, M. Herrler, R. J. Youle, and M. T. Fuller, "Mitofusin-1 protein is a generally expressed mediator of mitochondrial fusion in mammalian cells," Journal of Cell Science, vol. 116, no. 13, pp. 2763-2774, 2003.

[77] Z. Li, K. I. Okamoto, Y. Hayashi, and M. Sheng, "The importance of dendritic mitochondria in the morphogenesis and plasticity of spines and synapses," Cell, vol. 119, no. 6, pp. 873-887, 2004.

[78] T. Ono, K. Isobe, K. Nakada, and J. I. Hayashi, "Human cells are protected from mitochondrial dysfunction by complementation of DNA products in fused mitochondria," Nature Genetics, vol. 28, no. 3, pp. 272-275, 2001.

[79] P. A. Parone, S. Da Druz, D. Tondera et al., "Preventing mitochondrial fission impairs mitochondrial function and leads to loss of mitochondrial DNA," PLoS One, vol. 3, no. 9, Article ID e3257, 2008.

[80] D. T. W. Chang and I. J. Reynolds, "Differences in mitochondrial movement and morphology in young and mature primary cortical neurons in culture," Neuroscience, vol. 141, no. 2, pp. 727-736, 2006.

[81] H. Braak, K. Del Tredici, U. Rüb, R. A. I. De Vos, E. N. H. Jansen Steur, and E. Braak, "Staging of brain pathology related to sporadic Parkinson's disease," Neurobiology of Aging, vol. 24, no. 2, pp. 197-211, 2003.

[82] H. Braak, E. Ghebremedhin, U. Rüb, H. Bratzke, and K. Del Tredici, "Stages in the development of Parkinson's diseaserelated pathology," Cell and Tissue Research, vol. 318, no. 1, pp. 121-134, 2004.

[83] C. L. Liang, T. T. Wang, K. Luby-Phelps, and D. C. German, "Mitochondria mass is low in mouse substantia nigra dopamine neurons: implications for Parkinson's disease," Experimental Neurology, vol. 203, no. 2, pp. 370-380, 2007.
[84] M. P. Mattson, "Mitochondrial regulation of neuronal plasticity," Neurochemical Research, vol. 32, no. 4-5, pp. 707-715, 2007.

[85] D. T. W. Chang, A. S. Honick, and I. J. Reynolds, "Mitochondrial trafficking to synapses in cultured primary cortical neurons," Journal of Neuroscience, vol. 26, no. 26, pp. 70357045, 2006.

[86] R. D. Vale, "The molecular motor toolbox for intracellular transport," Cell, vol. 112, no. 4, pp. 467-480, 2003.

[87] A. Varadi, L. I. Johnson-Cadwell, V. Cirulli, Y. Yoon, V. J. Allan, and G. A. Rutter, "Cytoplasmic dynein regulates the subcellular distribution of mitochondria by controlling the recruitment of the fission factor dynamin-related protein-1," Journal of Cell Science, vol. 117, no. 19, pp. 4389-4400, 2004.

[88] K. J. De Vos, V. J. Allan, A. J. Grierson, and M. P. Sheetz, "Mitochondrial function and actin regulate dynamin-related protein 1-dependent mitochondrial fission," Current Biology, vol. 15, no. 7, pp. 678-683, 2005.

[89] A. F. MacAskill, J. E. Rinholm, A. E. Twelvetrees et al., "Miro1 is a calcium sensor for glutamate receptor-dependent localization of mitochondria at synapses," Neuron, vol. 61, no. 4, pp. 541-555, 2009.

[90] X. Wang and T. L. Schwarz, "The mechanism of Ca2+ -dependent regulation of kinesin-mediated mitochondrial motility," Cell, vol. 136, no. 1, pp. 163-174, 2009.

[91] M. Saotome, D. Safiulina, G. Szabadkai et al., "Bidirectional Ca2+-dependent control of mitochondrial dynamics by the Miro GTPase," Proceedings of the National Academy of Sciences of the United States of America, vol. 105, no. 52, pp. 20728-20733, 2008.

[92] X. Guo, G. T. Macleod, A. Wellington et al., "The GTPase dMiro is required for axonal transport of mitochondria to drosophila synapses," Neuron, vol. 47, no. 3, pp. 379-393, 2005.

[93] I. Kim, S. Rodriguez-Enriquez, and J. J. Lemasters, "Selective degradation of mitochondria by mitophagy," Archives of Biochemistry and Biophysics, vol. 462, no. 2, pp. 245-253, 2007.

[94] R. Scherz-Shouval, E. Shvets, E. Fass, H. Shorer, L. Gil, and Z. Elazar, "Reactive oxygen species are essential for autophagy and specifically regulate the activity of Atg4," EMBO Journal, vol. 26, no. 7, pp. 1749-1760, 2007.

[95] L. C. Gomes and L. Scorrano, "High levels of Fis1, a profission mitochondrial protein, trigger autophagy," Biochimica et Biophysica Acta, vol. 1777, no. 7-8, pp. 860-866, 2008.

[96] D. Narendra, A. Tanaka, D. F. Suen, and R. J. Youle, "Parkin is recruited selectively to impaired mitochondria and promotes their autophagy," Journal of Cell Biology, vol. 183, no. 5, pp. 795-803, 2008.

[97] D. Narendra, A. Tanaka, D. F. Suen, and R. J. Youle, "Parkin-induced mitophagy in the pathogenesis of Parkinson disease," Autophagy, vol. 5, no. 5, pp. 706-708, 2009.

[98] C. Vives-Bauza, C. Zhou, Y. Huang et al., "PINK1-dependent recruitment of Parkin to mitochondria in mitophagy," Proceedings of the National Academy of Sciences of the United States of America, vol. 107, no. 1, pp. 378-383, 2010.

[99] S. Kawajiri, S. Saiki, S. Sato et al., "PINK1 is recruited to mitochondria with parkin and associates with LC3 in mitophagy," FEBS Letters, vol. 584, no. 6, pp. 1073-1079, 2010.

[100] D. P. Narendra, S. M. Jin, A. Tanaka et al., "PINK1 is selectively stabilized on impaired mitochondria to activate Parkin," PLoS Biology, vol. 8, no. 1, Article ID e1000298, 2010. 
[101] S. Geisler, K. M. Holmström, D. Skujat et al., "PINK1/Parkinmediated mitophagy is dependent on VDAC1 and p62/SQSTM1," Nature Cell Biology, vol. 12, no. 2, pp. 119-131, 2010.

[102] M. E. Gegg, J. M. Cooper, K.-Y. Chau, M. Rojo, A. H. V. Schapira, and J.-W. Taanman, "Mitofusin 1 and mitofusin 2 are ubiquitinated in a PINK1/parkin-dependent manner upon induction of mitophagy," Human Molecular Genetics, vol. 19, no. 24, pp. 4861-4870, 2010.

[103] J. Alegre-Abarrategui, H. Christian, M. M. P. Lufino et al., "LRRK2 regulates autophagic activity and localizes to specific membrane microdomains in a novel human genomic reporter cellular model," Human Molecular Genetics, vol. 18, no. 21, pp. 4022-4034, 2009.

[104] E. D. Plowey, S. J. Cherra, Y. J. Liu, and C. T. Chu, "Role of autophagy in G2019S-LRRK2-associated neurite shortening in differentiated SH-SY5Y cells," Journal of Neurochemistry, vol. 105, no. 3, pp. 1048-1056, 2008.

[105] C. T. Chu, J. Zhu, and R. Dagda, "Beclin 1-independent pathway of damage-induced mitophagy and autophagic stress: implications for neurodegeneration and cell death," Autophagy, vol. 3, no. 6, pp. 663-666, 2007.

[106] S. J. Cherra and C. T. Chu, "Autophagy in neuroprotection and neurodegeneration: a question of balance," Future Neurology, vol. 3, no. 3, pp. 309-323, 2008.

[107] J. P. de Mattos, A. Alencar, A. G. da Silva, and M. E. Fonseca, "Ultrastructural aspects of Parkinson disease," Arquivos de Neuro-Psiquiatria, vol. 47, no. 4, pp. 430-437, 1989.

[108] P. A. Trimmer, R. H. Swerdlow, J. K. Parks et al., "Abnormal mitochondrial morphology in sporadic Parkinson's and Alzheimer's disease cybrid cell lines," Experimental Neurology, vol. 162, no. 1, pp. 37-50, 2000.

[109] M. J. Barsoum, H. Yuan, A. A. Gerencser et al., "Nitric oxideinduced mitochondrial fission is regulated by dynaminrelated GTPases in neurons," EMBO Journal, vol. 25, no. 16, pp. 3900-3911, 2006.

[110] N. A. Pham, T. Richardson, J. Cameron, B. Chue, and B. H. Robinson, "Altered mitochondrial structure and motion dynamics in living cells with energy metabolism defects revealed by real time microscope imaging," Microscopy and Microanalysis, vol. 10, no. 2, pp. 247-260, 2004.

[111] M. K. Borland, K. P. Mohanakumar, J. D. Rubinstein et al., "Relationships among molecular genetic and respiratory properties of Parkinson's disease cybrid cells show similarities to Parkinson's brain tissues," Biochimica et Biophysica Acta, vol. 1792, no. 1, pp. 68-74, 2009.

[112] G. Cappelletti, T. Surrey, and R. Maci, "The parkinsonism producing neurotoxin MPP affects microtubule dynamics by acting as a destabilising factor," FEBS Letters, vol. 579, no. 21, pp. 4781-4786, 2005.

[113] Y. Ren and J. Feng, "Rotenone selectively kills serotonergic neurons through a microtubule-dependent mechanism," Journal of Neurochemistry, vol. 103, no. 1, pp. 303-311, 2007.

[114] A. R. Esteves, D. M. Arduino, R. H. Swerdlow, C. R. Oliveira, and S. M. Cardoso, "Oxidative Stress involvement in alphasynuclein oligomerization in Parkinsons disease cybrids," Antioxid Redox Signal, vol. 11, no. 3, pp. 439-448, 2009.

[115] M. K. Borland, P. A. Trimmer, J. D. Rubinstein et al., "Chronic, low-dose rotenone reproduces lewy neurites found in early stages of Parkinson's disease, reduces mitochondrial movement and slowly kills differentiated SH-SY5Y neural cells," Molecular Neurodegeneration, vol. 3, no. 1, Article ID 21, 2008.
[116] J. C. Greene, A. J. Whitworth, I. Kuo, L. A. Andrews, M. B. Feany, and L. J. Pallanck, "Mitochondrial pathology and apoptotic muscle degeneration in Drosophila parkin mutants," Proceedings of the National Academy of Sciences of the United States of America, vol. 100, no. 7, pp. 4078-4083, 2003.

[117] C. Wang, R. Lu, X. Ouyang et al., "Drosophila overexpressing parkin $\mathrm{R} 275 \mathrm{~W}$ mutant exhibits dopaminergic neuron degeneration and mitochondrial abnormalities," Journal of Neuroscience, vol. 27, no. 32, pp. 8563-8570, 2007.

[118] M. G. Riparbelli and G. Callaini, "The Drosophila parkin homologue is required for normal mitochondrial dynamics during spermiogenesis," Developmental Biology, vol. 303, no. 1, pp. 108-120, 2007.

[119] J. J. Palacino, D. Sagi, M. S. Goldberg et al., "Mitochondrial dysfunction and oxidative damage in parkin-deficient mice," Journal of Biological Chemistry, vol. 279, no. 18, pp. 1861418622, 2004.

[120] C. C. Stichel, X. R. Zhu, V. Bader, B. Linnartz, S. Schmidt, and H. Lübbert, "Mono- and double-mutant mouse models of Parkinson's disease display severe mitochondrial damage," Human Molecular Genetics, vol. 16, no. 20, pp. 2377-2393, 2007.

[121] H. Mortiboys, K. J. Thomas, W. J. H. Koopman et al., "Mitochondrial function and morphology are impaired in parkin-mutant fibroblasts," Annals of Neurology, vol. 64, no. 5, pp. 555-565, 2008.

[122] M. Muftuoglu, B. Elibol, O. Dalmizrak et al., "Mitochondrial complex I and IV activities in leukocytes from patients with parkin mutations," Movement Disorders, vol. 19, no. 5, pp. 544-548, 2004.

[123] Y. Kuroda, T. Mitsui, M. Kunishige et al., "Parkin enhances mitochondrial biogenesis in proliferating cells," Human Molecular Genetics, vol. 15, no. 6, pp. 883-895, 2006.

[124] F. Darios, O. Corti, C. B. Lücking et al., "Parkin prevents mitochondrial swelling and cytochrome c release in mitochondria-dependent cell death," Human Molecular Genetics, vol. 12, no. 5, pp. 517-526, 2003.

[125] C. A. Gautier, T. Kitada, and J. Shen, "Loss of PINK1 causes mitochondrial functional defects and increased sensitivity to oxidative stress," Proceedings of the National Academy of Sciences of the United States of America, vol. 105, no. 32, pp. 11364-11369, 2008.

[126] I. E. Clark, M. W. Dodson, C. Jiang et al., "Drosophila pink1 is required for mitochondrial function and interacts genetically with parkin," Nature, vol. 441, no. 7097, pp. 11621166, 2006.

[127] J. Park, C. I. Yoo, C. S. Sim et al., "A retrospective cohort study of Parkinson's disease in Korean shipbuilders," NeuroToxicology, vol. 27, no. 3, pp. 445-449, 2006.

[128] Y. Yang, S. Gehrke, Y. Imai et al., "Mitochondrial pathology and muscle and dopaminergic neuron degeneration caused by inactivation of Drosophila Pink1 is rescued by Parkin," Proceedings of the National Academy of Sciences of the United States of America, vol. 103, no. 28, pp. 10793-10798, 2006.

[129] M. E. Haque, K. J. Thomas, C. D’Souza et al., "Cytoplasmic Pink1 activity protects neurons from dopaminergic neurotoxin MPTP," Proceedings of the National Academy of Sciences of the United States of America, vol. 105, no. 5, pp. 1716-1721, 2008.

[130] C. Zhou, Y. Huang, Y. Shao et al., "The kinase domain of mitochondrial PINK1 faces the cytoplasm," Proceedings of the 
National Academy of Sciences of the United States of America, vol. 105, no. 33, pp. 12022-12027, 2008.

[131] A. Weihofen, K. J. Thomas, B. L. Ostaszewski, M. R. Cookson, and D. J. Selkoe, "Pink1 forms a multiprotein complex with miro and milton, linking Pink1 function to mitochondrial trafficking," Biochemistry, vol. 48, no. 9, pp. 2045-2052, 2009.

[132] S. Geisler, K. M. Holmström, A. Treis et al., "The PINK1/ Parkin-mediated mitophagy is compromised by PDassociated mutations," Autophagy, vol. 6, no. 7, pp. 871-878, 2010.

[133] Y. Yang, Y. Ouyang, L. Yang et al., "Pink1 regulates mitochondrial dynamics through interaction with the fission/fusion machinery," Proceedings of the National Academy of Sciences of the United States of America, vol. 105, no. 19, pp. 7070-7075, 2008.

[134] P. A. Trimmer, M. K. Borland, P. M. Keeney, J. P. Bennett, and W. D. Parker, "Parkinson's disease transgenic mitochondrial cybrids generate Lewy inclusion bodies," Journal of Neurochemistry, vol. 88, no. 4, pp. 800-812, 2004.

[135] R. K. Dagda, S. J. Cherra, S. M. Kulich, A. Tandon, D. Park, and C. T. Chu, "Loss of PINK1 function promotes mitophagy through effects on oxidative stress and mitochondrial fission," Journal of Biological Chemistry, vol. 284, no. 20, pp. 13843-13855, 2009.

[136] A. Sandebring, K. J. Thomas, A. Beilina et al., "Mitochondrial alterations in PINK1 deficient cells are influenced by calcineurin-dependent dephosphorylation of dynaminrelated protein 1," PLoS One, vol. 4, no. 5, Article ID e5701, 2009.

[137] M. E. Gegg, J. M. Cooper, A. H. V. Schapira, and J. W. Taanman, "Silencing of PINK1 expression affects mitochondrial DNA and oxidative phosphorylation in DOPAMINERGIC cells," PLoS One, vol. 4, no. 3, Article ID e4756, 2009.

[138] A. Wood-Kaczmar, S. Gandhi, Z. Yao et al., "PINK1 is necessary for long term survival and mitochondrial function in human dopaminergic neurons," PLoS One, vol. 3, no. 6, Article ID e2455, 2008.

[139] C. T. Chu, "Tickled PINK1: mitochondrial homeostasis and autophagy in recessive Parkinsonism," Biochimica et Biophysica Acta, vol. 1802, no. 1, pp. 20-28, 2010.

[140] R. Ichishita, K. Tanaka, Y. Sugiura, T. Sayano, K. Mihara, and T. Oka, "An RNAi screen for mitochondrial proteins required to maintain the morphology of the organelle in Caenorhabditis elegans," Journal of Biochemistry, vol. 143, no. 4, pp. 449-454, 2008.

[141] P. Anglade, S. Vyas, F. Javoy-Agid et al., "Apoptosis and autophagy in nigral neurons of patients with Parkinson's disease," Histology and Histopathology, vol. 12, no. 1, pp. 2531, 1997.

[142] T. Vogiatzi, M. Xilouri, K. Vekrellis, and L. Stefanis, "Wild type $\alpha$-synuclein is degraded by chaperone-mediated autophagy and macroautophagy in neuronal cells," Journal of Biological Chemistry, vol. 283, no. 35, pp. 23542-23556, 2008.

[143] A. R. Winslow, C.-W. Chen, S. Corrochano et al., " $\alpha$ synuclein impairs macroautophagy: implications for Parkinson's disease," Journal of Cell Biology, vol. 190, no. 6, pp. 1023-1037, 2010.

[144] D. M. Arduíno, A. R. Esteves, C. R. Oliveira, and S. M. Cardoso, "Mitochondrial metabolism modulation: a new therapeutic approach for Parkinson's disease," CNS and Neurological Disorders Drug Targets, vol. 9, no. 1, pp. 105119, 2010.
[145] A. R. Esteves, D. M. Arduino, R. H. Swerdlow, C. R. Oliveira, and S. M. Cardoso, "Microtubule depolymerization potentiates alpha-synuclein oligomerization," Frontiers in Aging Neuroscience, vol. 1, article 5, 2010.

[146] E. Perlson, S. Maday, M. M. Fu, A. J. Moughamian, and E. L. F. Holzbaur, "Retrograde axonal transport: pathways to cell death?" Trends in Neurosciences, vol. 33, no. 7, pp. 335-344, 2010.

[147] A. R. Esteves, D. M. Arduíno, R. H. Swerdlow, C. R. Oliveira, and S. M. Cardoso, "Dysfunctional mitochondria uphold calpain activation: contribution to Parkinson's disease pathology," Neurobiology of Disease, vol. 37, no. 3, pp. 723-730, 2010.

[148] M. Navratil, A. Terman, and E. A. Arriaga, "Giant mitochondria do not fuse and exchange their contents with normal mitochondria," Experimental Cell Research, vol. 314, no. 1, pp. 164-172, 2008.

[149] A. Jahani-Asl, K. Pilon-Larose, W. Xu et al., "The mitochondrial inner membrane GTPase, Optic Atrophy 1 (Opa1), restores mitochondrial morphology and promotes neuronal survival following excitotoxicity," The Journal of Biological Chemistry, vol. 286, no. 6, pp. 4772-4782, 2011. 


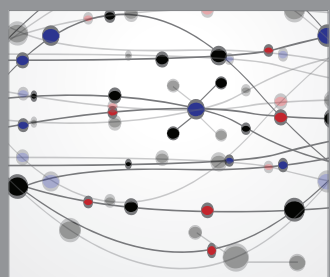

The Scientific World Journal
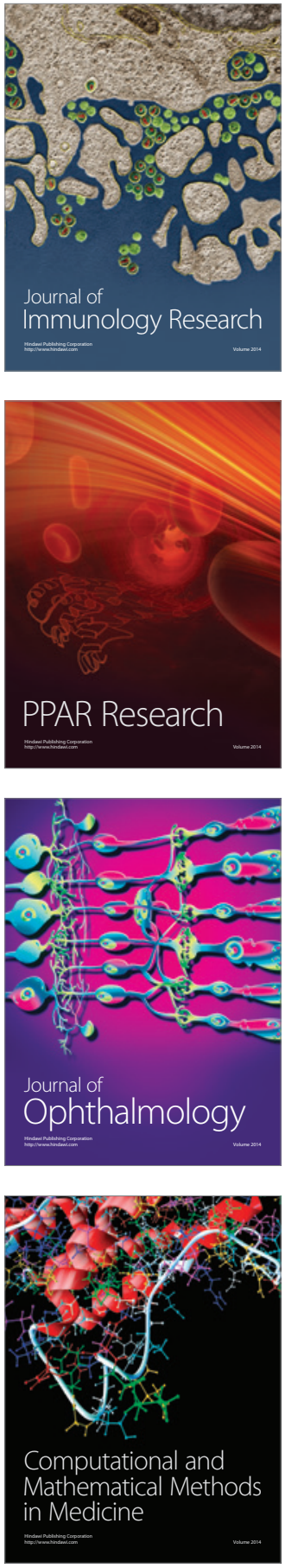

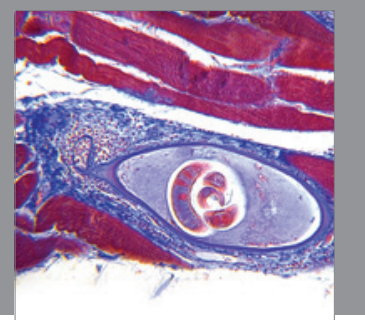

Gastroenterology

Research and Practice
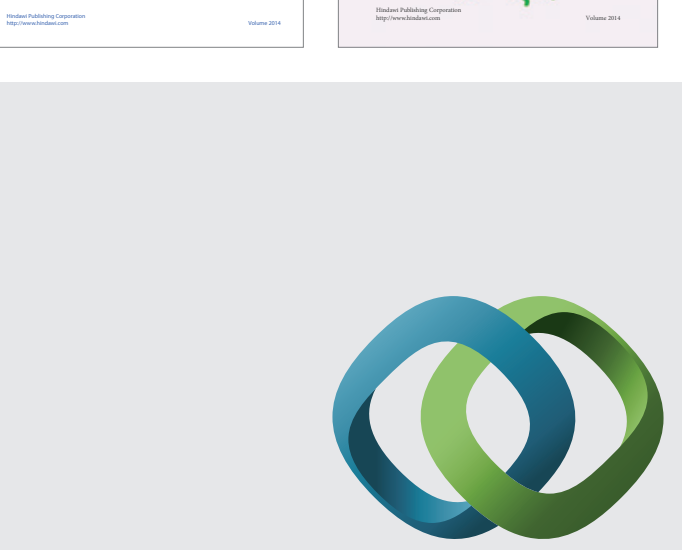

\section{Hindawi}

Submit your manuscripts at

http://www.hindawi.com
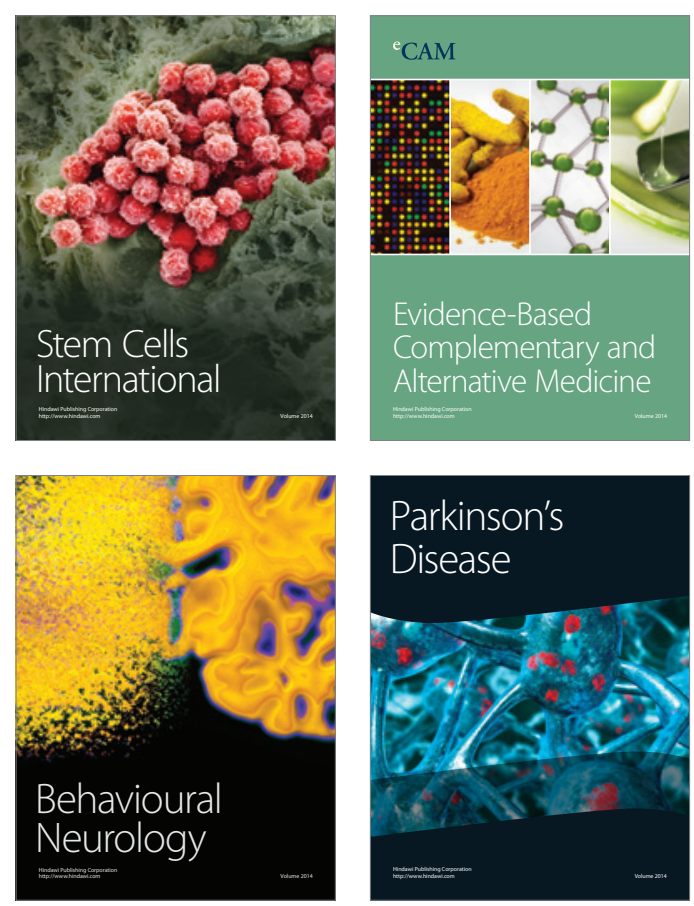

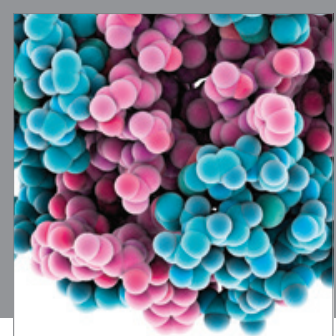

Journal of
Diabetes Research

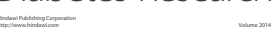

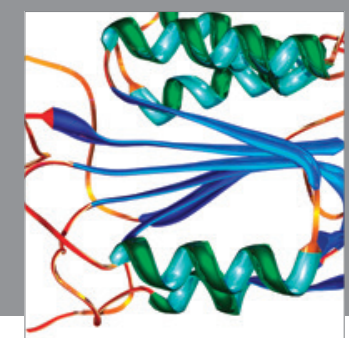

Disease Markers
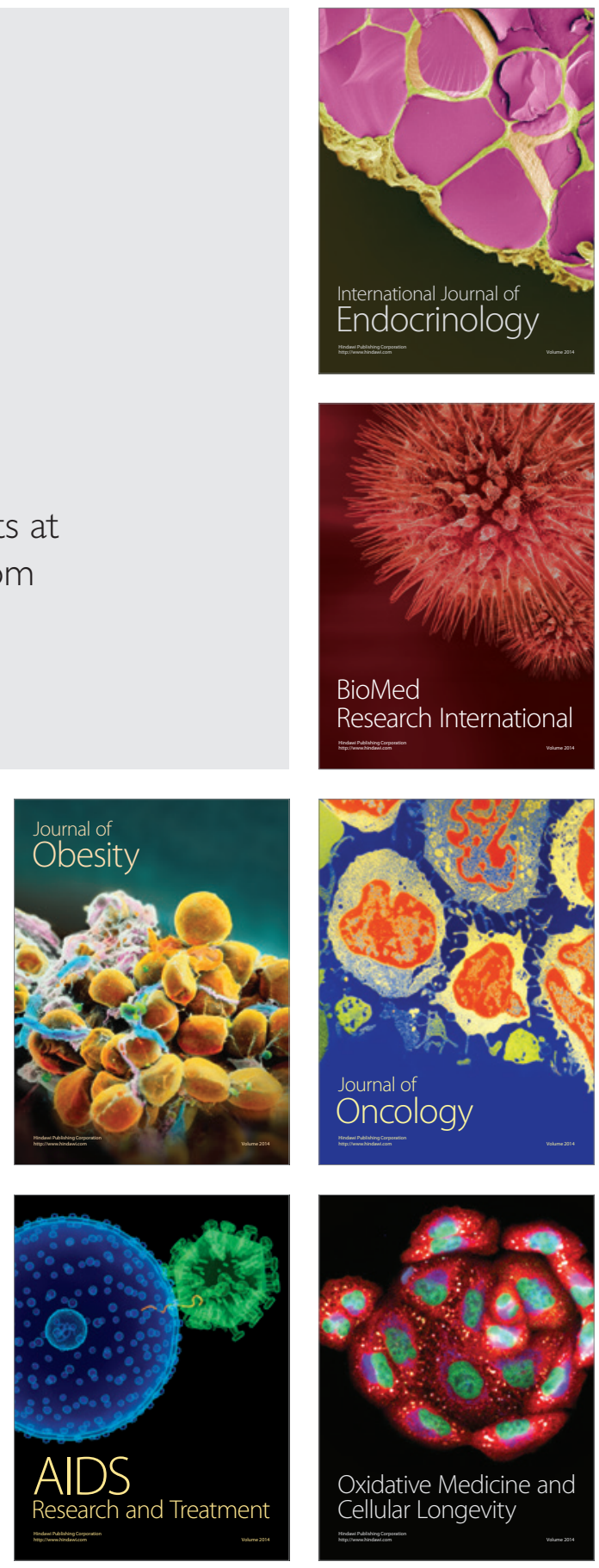